\title{
DETERMINAÇÃO DA COR, IMAGEM SUPERFICIAL TOPOGRÁFICA E ÂNGULO DE CONTATO DE BIOFILMES DE DIFERENTES FONTES DE AMIDO ${ }^{1}$
}

\author{
Determination of color, topographic superficial image and contact angle of the \\ biofilms of different starch sources
}

\author{
Washington Azevêdo da Silva², Joelma Pereira ${ }^{3}$, Carlos Wanderlei Piler de Carvalho ${ }^{4}$, \\ Fabiana Queiroz Ferrua ${ }^{5}$
}

\begin{abstract}
RESUMO
Atualmente, um dos problemas que acometem o meio ambiente é a poluição ocasionada pelo descarte de filmes plásticos de polímeros sintéticos sem nenhum controle. Uma das alternativas para minimizar este problema é a utilização de amido na elaboração de materiais biodegradáveis que possuem alta degradabilidade quando lançados no meio ambiente. Apesar do número crescente de trabalhos de pesquisas utilizando diferentes materiais biodegradáveis, o conhecimento das propriedades físicas de biofilmes elaborados com amido ainda é bastante reduzido. Objetivou-se com este trabalho determinar a diferença de cor, a imagem superficial topográfica por microscopia eletrônica de varredura e o ângulo de contato de biofilmes elaborados com três diferentes tipos de amido: filme de fécula de batata (FFB), filme de fécula de mandioca (FFM) e filme de amido de milho (FAM) com três concentrações: 1, 2 e 3\% empregando-se um delineamento inteiramente casualizado com três repetições. A interação concentração e tipo de amido foi estatisticamente significativa para ângulo de contato e não significativa para a diferença de cor. A diferença de cor foi afetada pela fonte de amido e o maior valor observado para filme de fécula de mandioca. Com o aumento da concentração de amido, o ângulo de contato diminuiu para filmes de fécula de mandioca e de batata, diferentemente para os biofilmes de milho, onde esta relação foi constante.
\end{abstract}

Termos para indexação: Biofilmes, amido, cor, microscopia, ângulo de contato.

\begin{abstract}
Nowadays, the pollution has considerably increased by the large amount of the non-degradable synthetic plastics that is discarded everyday in the environment. One of the alternatives that could contribute to minimize this problem would be the use of the starch as main composite in biodegradable films also called biofilms; in other words, this material is easily degradable when disposed in the environment. Despite the growing number of the scientific papers there is a lack of knowledge of the physical properties of starch in biofilms. The aim of this work was to study the color variation, the topographic superficial surface by scanning electron microscopy and the water absorption by contact angle of biofilms produced with three different starch sources: potato starch film (PSF), cassava starch film (CSF) and maize starch film (MSF) with varied concentration: 1, 2 and 3\% to prepare biofilms using a complete randomize design with three repetitions. The interaction between starch source and concentration was statistically significant for contact angle whereas it was not significant for color. The color difference was affected by starch source being higher for CSF. As concentration increased in biofilms of potato and cassava, the contact angle decreased in contrast to maize biofilms which remained constant.
\end{abstract}

Index terms: Biofilms, starch, color, microscopy, contact angle.

(Recebido para publicação em 23 de março de 2005 e aprovado em 7 de outubro de 2005)

\section{INTRODUÇÃO}

As preocupações ambientais associadas à grande disposição de materiais plásticos têm proporcionado a investigação de alternativas solucionadoras em substituição ao plástico comum, principalmente no tocante à biodegradabilidade destes materiais. No Brasil, cerca de 240 mil toneladas de lixo são produzidas por dia, e a maior parte do lixo tem como destino os lixões a céu aberto, constituindo um sério problema sanitário (CEREDA et al., 2003). A adoção de programas de coleta seletiva e reciclagem é uma das alternativas e a produção de materiais biodegradáveis a partir de fontes renováveis é considerada uma forma de reciclagem (POOVARODOM \& PRADITDOUNG, 2003). Dessa forma, várias pesquisas com diferentes materiais (amido, celulose e lipídios) têm sido desenvolvidas na tentativa de minimizar, diminuir ou

\footnotetext{
Parte do trabalho de dissertação de Mestrado do primeiro autor.

²Engenheiro Agrônomo, Mestre - Departamento de Ciência dos Alimentos/DCA - Universidade Federal de Lavras/UFLA - Cx. P. 3037 - 37.200-000 Lavras, MG - was@vicosa.ufv.br

${ }^{3}$ Engenheira Agrônoma, Professora Adjunta do Departamento de Ciência dos Alimentos/DCA - Universidade Federal de Lavras/UFLA - Cx. P. 3037 37.200-000 - Lavras, MG - joper@ufla.br

${ }^{4}$ Engenheiro Agrônomo, Pesquisador,Ph.D. - Embrapa Alimentos - cwpiler@ctaa.embrapa.br

${ }^{5}$ Engenheira de Alimentos, Professora. Adjunta - Departamento de Ciência dos Alimentos/DCA - Universidade Federal de Lavras/UFLA Cx. P. 3037 - 37.200-000 - Lavras, MG - fqueiroz@ufla.br
} 
solucionar o problema da poluição através da obtenção de plásticos biodegradáveis, ou seja, os biofilmes. O amido tem recebido especial atenção e as pesquisas com este polímero se intensificaram nos últimos anos, pois o amido possui baixo custo, abundância e alta aplicabilidade (BENGTSSON et al., 2003).

Martins (2003) lembra que os biofilmes podem ser produzidos a partir de polissacarídeos e proteínas cujas cadeias são longas e capazes de produzir matrizes contínuas que dão estrutura ao filme. Mali et al. (2004) citam que o uso do amido na produção de plásticos iniciouse nos anos 70 e os primeiros estudos sobre o uso do amido em embalagens biodegradáveis foram baseados na substituição parcial da matriz polimérica sintética pelo amido. A fécula de mandioca tem sido empregada na elaboração e obtenção de biofilmes, mas pouco se sabe das propriedades físicas desses (CEREDA et al., 1992; LAROTONDA et al., 2004). Já os amidos de batata e de milho são fontes outras que também têm sido pesquisadas na obtenção de filmes biodegradáveis (BENGTSSON et al., 2003; JANSSON \& THUVANDER, 2004; THIRÉ et al., 2003). De acordo com Oliveira \& Cereda (1999), o amido quando geleificado possui a propriedade de formar géis, e esses quando desidratados originam películas rígidas e transparentes, brilhantes, resistentes semelhantes às películas de celulose. A obtenção dos biofilmes de amido baseia-se na gelatinização com posterior retrogradação. A temperatura de gelatinização varia de acordo com a fonte de amido. A fécula de batata: 56 a $66^{\circ} \mathrm{C}$, fécula de mandioca: 62 a $66^{\circ} \mathrm{C}$ e amido de milho de 56 a $72^{\circ} \mathrm{C}$ (CIACCO \& CRUZ, 1982). Para Lawton (1996), os filmes de amido puro apresentam diferentes propriedades quando elaborados com diferentes tipos de amido. Segundo Sobral (2000), as propriedades dos biofilmes dependem do biopolímero usado, das condições de fabricação e das condições ambientais que são importantes fatores por causa da natureza higroscópica dos biopolímeros.

A cor é um atributo de fundamental importância para um produto. Poucos são os trabalhos que relatam a determinação da cor para biofilmes, e quando determinada utiliza-se o sistema $L^{*} a * b *$ em que $L^{*}$ varia de preto a branco (0 a 100), a* varia do verde ao vermelho $(-60 \mathrm{a}+60)$ e b* varia de azul a amarelo $(-60 \mathrm{a}+60)$ (ROY et al., 1999; SOBRAL et al., 2000). As técnicas microscópicas têm sido bastante utilizadas para a análise das imagens de biofilmes de amido e de produtos amiláceos, de modo geral (LAROTONDA et al., 2004; SHANDERA \& JACKSON, 2002; THIRÉ et al., 2004; WATANABE et al., 2003; WILHELM et al., 2003). Bengtsson et al. (2003) utilizaram a microscopia eletrônica de varredura no monitoramento da superfície de filmes à base de amido de batata. As fotomicrografias revelaram que os filmes apresentaram superfície muito lisa. Jayasekara et al. (2004) também utilizaram a microscopia eletrônica de varredura para investigar a superfície de biofilmes de amido de trigo e observaram a presença de trincas e rachaduras na superfície dos biofilmes.

Outro parâmetro que tem sido avaliado em biofilmes é o ângulo de contato conhecido também como ângulo de molhabilidade. $\mathrm{O}$ ângulo de contato representa o valor em graus que uma determinada substância líquida forma com a superfície, a exemplo, o ângulo formado de uma gota de água sobre a superfície de um filme. Os valores para ângulo de contato para biofilmes são dependentes da constituição do material entre outros, como: tipo de solvente, etc., (BRUNO et al., 1995; ABURTO et al., 1999; BENGTSSON et al., 2003). Jayasekara et al. (2004) citam que o ângulo de contato está correlacionado com a rugosidade da superfície dos biofilmes, e que este aumenta com o aumento da rugosidade. Bialopiotrowicz (2003) cita que os efeitos de solventes orgânicos sobre os géis de amido são essenciais, por exemplo, para determinação das propriedades dos filmes de amido, habilidade dos géis em serem firmes, secar, e manter a estrutura frente a solventes orgânicos não-aquosos. Outro ponto importante, é que filmes de amido têm sido desenvolvidos para serem utilizados como recobrimentos comestíveis de frutas, pois conferem brilho e melhor aparência aos frutos. Assim, frutas são armazenadas em câmaras-frias em que a umidade relativa que pode chegar a 95\% dependendo do produto. Dessa forma, o filme quando utilizado como recobrimento e submetido a tal condição poderia absorver água podendo perder a sua aplicabilidade. Logo, julga-se importante realizar o teste do ângulo de contato com o líquido, água. Portanto, com o presente trabalho, objetivou-se determinar o ângulo de contato, diferença de cor e a superfície dos biofilmes de amido de milho, fécula de batata e de fécula de mandioca.

\section{MATERIAL E MÉTODOS}

\section{Elaboração e identificação dos biofilmes}

As suspensões de amido a 1, 2 e $3 \%$ foram obtidas a partir da adição de 1, 2 e $3 \mathrm{~g}$ de amido de milho, fécula de batata e fécula de mandioca completando-se o volume para $100 \mathrm{~mL}$ com água destilada em béquer.

As suspensões padronizadas foram agitadas homogeneamente e lentamente aquecidas até $70^{\circ} \mathrm{C}$ com o objetivo de se obter a gelatinização do amido. Os géis 
foram mantidos nesta temperatura por cinco minutos. $\mathrm{O}$ gel obtido foi resfriado até atingir a temperatura ambiente, o que ocorreu em cerca de duas horas. Após o resfriamento dos géis, alíquotas de $10 \mathrm{~mL}$ foram tomadas e colocadas dentro de placas de poliestireno cristal, não autoclavável com $110 \mathrm{~mm}$ de diâmetro e $15 \mathrm{~mm}$ de espessura, de modo que toda a superfície interna da placa fosse coberta uniformemente com o gel. As placas contendo os géis foram colocadas em sala à temperatura ambiente de $25^{\circ} \mathrm{C}\left( \pm 2^{\circ} \mathrm{C}\right)$ e umidade relativa de $50 \%$ ( $\pm 5 \%$ UR). Após 24 horas, os filmes foram retirados facilmente das placas e armazenados em sacos de polietileno de baixa densidade, também a mesma temperatura e umidade relativa citadas acima. Para realização das análises de caracterização dos biofilmes, estes receberam a seguinte identificação: FAM1 - Filme de amido de milho a 1\%; FAM2 - Filme de amido de milho a 2\%; FAM3 - Filme de amido de milho a 3\%; FFB1- Filme de fécula de batata a $1 \%$; FFB2- Filme de fécula de batata a $2 \%$; FFB3- Filme de fécula de batata a 3\%; FFM1- Filme de fécula de mandioca a $1 \%$; FFM2- Filme de fécula de mandioca a 2\%; FFM3- Filme de fécula de mandioca a $3 \%$.

$\mathrm{O}$ valor médio da espessura dos biofilmes foi obtido através de leitura com paquímetro manual e expressa em mm: FFB1 -0,10; FFB2-0,10; FFB3 - 0, 10; FFM1 -0,09; FFM2 - 0,09; FFM3 - 0,10; FAM1 - 0,11; FAM2 - 0,11; FAM3 - 0,11.

\section{Diferença de cor}

A cor dos filmes foi determinada de acordo com a metodologia proposta por Gennadios et al. (1996). Os valores de $\mathrm{L}^{*}, \mathrm{a}^{*} \mathrm{e} \mathrm{b}^{*}$ foram determinados com aparelho colorímetro Minolta modelo CR 400 trabalhando com $\mathrm{D}_{65}$ (luz do dia) e usando-se os padrões CIE Lab. Os filmes foram colocados sobre uma superfície branca (papel) e procedeu-se às leituras. Três leituras foram realizadas em cada amostra de filme e com três repetições. A diferença de cor (DE*) foi obtida através da equação 1 :

$$
\Delta \mathrm{E}^{*}=\left[\left(\Delta \mathrm{L}^{*}\right)^{2}+\left(\Delta \mathrm{a}^{*}\right)^{2}+\left(\Delta \mathrm{b}^{*}\right)^{2}\right]^{0,5}
$$

em que: $\mathrm{DE}^{*}=$ valor para a diferença de cor; Padrão branco: $L^{*}=92,40 ; a^{*}=0,3162 ; b^{*}=0,3326$ padrão branco; $D^{*}=$ diferença entre $\mathrm{L}^{*}$ padrão branco e $\mathrm{L}^{*}$ da amostra; $\mathrm{Da}^{*}=$ diferença entre o $a^{*}$ padrão branco e o $a^{*}$ da amostra; $\mathrm{Db}^{*}$ $=$ diferença entre o $b^{*}$ padrão branco e o $b^{*}$ da amostra. A análise foi realizada no Laboratório de Grãos e Cereais, do Departamento de Ciência dos Alimentos, da Universidade Federal de Lavras - UFLA.

\section{Microscopia eletrônica de varredura}

A análise foi realizada no Laboratório de UltraEstrutura e Microscopia Eletrônica do Departamento de Fitopatologia da UFLA. A obtenção das fotomicrografias dos filmes foi realizada de acordo com a metodologia proposta por Bengtsson et al. (2003). Foi utilizado microscópio eletrônico de varredura (modelo EVO 40, marca LEQ), onde, pequenas amostras dos filmes ( 2 $\mathrm{mm}$ ) foram acondicionadas em "stubs" e banhadas com ouro, processo denominado de metalização, fixadas no aparelho e fotomicrografadas. Tensão de aceleração de $20 \mathrm{KV}$ e ampliação de 5000x.

\section{Ângulo de contato}

A análise foi realizada no Laboratório do Instituto de Macromoléculas Professora Eloísa Mano do Centro de Tecnologia da Universidade Federal do Rio de Janeiro UFRJ. As medidas de ângulo de contato foram obtidas empregando-se o goniômetro Ramé-Hart, modelo NRL, operado em ar e a temperatura ambiente. Os ângulos de contato do lado direito e esquerdo da gota foram calculados automaticamente por meio de software RHI 2001 Imaging Software instalado em um computador acoplado ao equipamento. Foram realizadas medidas de ângulo do lado direito e esquerdo de três gotas. Amostras de $30 \mathrm{~mm}$ de comprimento e $5 \mathrm{~mm}$ de largura foram fixadas em uma lâmina de vidro por meio de fita adesiva dupla face. As lâminas foram colocadas na base do aparelho. Uma gota de água de $0,1 \mathrm{~mL}$ foi colocada sobre a superfície da amostra com auxílio de uma seringa e a imagem da gota foi captada por uma câmera digital a intervalo de $0,5 \mathrm{~s}$. Os valores do ângulo de contato representam a média dos ângulos das três repetições que foram obtidos por somatória e divisão do ângulo direito e esquerdo de cada gota com a superfície do filme.

\section{Delineamento experimental e análise estatística}

Foi utilizado o delineamento inteiramente casualizado (DIC), com três repetições. Os tratamentos foram dispostos em esquema fatorial ( $3 \times 3$ ), sendo constituídos pelas combinações das fontes de amido: amido de milho, fécula de batata e de fécula de mandioca com as concentrações de amido: 1, 2 e 3\%. Os resultados para diferença de cor e ângulo de contato foram submetidos à análise de variância, de acordo com o esquema fatorial. Foi utilizado programa SISVAR Versão 4.3 (FERREIRA, 2003). A análise de regressão foi utilizada para avaliação dos parâmetros em função da concentração de amido, e o teste de Tukey (5\%) para comparação dos diferentes tipos de amido. 


\section{RESULTADOS E DISCUSSÃO}

Na Tabela 1, apresenta-se a análise de variância (ANOVA) para os parâmetros diferença de cor e ângulo de contato.

\section{Diferença de cor}

Em geral, os filmes obtidos com amido de milho, fécula de batata e fécula de mandioca em todas as concentrações testadas apresentaram-se transparentes e brilhantes. Verificouse efeito significativo das fontes de amido sobre a diferença de cor dos filmes e não-significativo para concentração e interação entre concentração e tipo de amido (Tabela 1). Na Tabela 2, apresentam-se as médias para a diferença de cor em função das diferentes fontes de amido, na qual pode ser verificado que os filmes de fécula de mandioca apresentaram maior coloração que os filmes de amido de milho (FAM) e filmes de fécula de batata (FFB), independente da concentração utilizada. É importante ressaltar que durante o processo de gelatinização do amido ocorre mudança na coloração devido à perda da estrutura e cristalinidade dos grânulos de amido, tornando-se gel com certa opacidade.

\section{Microscopia eletrônica de varredura}

Na Figura 1, apresentam-se as fotomicrografias dos filmes de amido de milho (FAM), nas concentrações de $1 \%$ (a), $2 \%$ (b) e $3 \%$ (c), respectivamente.

TABELA 1 - Análise de variância para os parâmetros diferença de cor e ângulo de contato de biofilmes em função do tipo e concentração de amido.

\begin{tabular}{lccc}
\hline \multicolumn{1}{c}{ Fontes de variação } & GL & \multicolumn{2}{c}{ Parâmetros avaliados } \\
\cline { 3 - 4 } & & Diferença de cor & \multicolumn{1}{c}{ Ângulo de contato } \\
\hline Tipo & 2 & $0,1896^{*}$ & $51,2344^{*}$ \\
Concentração & 2 & $0,0712 \mathrm{~ns}$ & $497,8711^{*}$ \\
Tipo x Concentração & 4 & $0,0088 \mathrm{~ns}$ & $1876,6338^{*}$ \\
Resíduo & 18 & 0,0176 & 0,4677 \\
CV $(\%)$ & & 4,1600 & 1,0100 \\
\hline
\end{tabular}

* significativo a $5 \%$ de probabilidade pelo teste de $\mathrm{F}$.

ns - não-significativo a $5 \%$ de probabilidade pelo teste de $\mathrm{F}$.

TABELA 2 - Quadro de médias $^{1}$ para a diferença de cor de biofilmes de amido de milho (FAM), fécula de batata (FFB) e fécula de mandioca (FFM).

\begin{tabular}{cc}
\hline Fontes de amido & Diferença de cor $\left(\Delta \mathbf{E}^{*}\right)$ \\
\hline FAM & $3,05 \mathrm{a}$ \\
FFB & $3,18 \mathrm{a}$ \\
FFM & $3,35 \mathrm{~b}$ \\
\hline
\end{tabular}

${ }^{1}$ Médias seguidas de mesma letra na coluna não diferiram entre si pelo teste de Tukey a $5 \%$ de probabilidade.





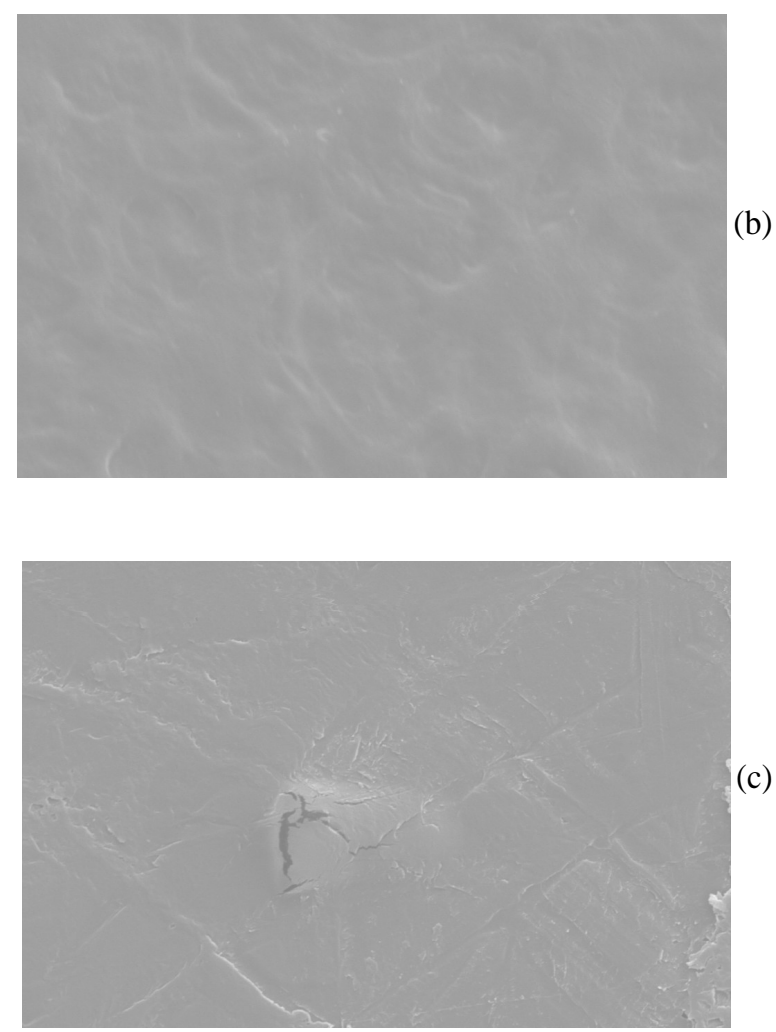

FIGURA 1 - Fotomicrografias dos biofilmes de amido de milho nas concentrações de 1\% (a), $2 \%$ (b) e 3\% (c).

As Figuras 1(a), 1(b) e 1(c) apresentam uma matriz contínua, variando o relevo da superfície de acordo com a concentração de amido de milho. Na Figura 1(c) (3\% de amido) observa-se o aparecimento de trincas ou rachaduras, o que pode ser atribuído a um maior grau de cristalização da matriz amilácea ocasionada durante a secagem do material e, ainda relacionada à temperatura de elaboração dos géis que foi de $70{ }^{\circ} \mathrm{C}$ e que provavelmente não foi suficiente para a gelatinização completa dos grânulos de amido de milho, já que a temperatura para completa gelatinização dos grânulos de amido de milho é superior a $70^{\circ} \mathrm{C}$. Para Rindlav-Westling et al. (2003), a presença de rugosidade na superfície de filmes de amido está relacionada com a separação de fase da amilose e amilopectina e, ainda, com a alta cristalinidade dos filmes. Na Figura 2 são apresentadas as fotomicrografias dos filmes de fécula de batata na concentração de $1 \%$ (a), $2 \%$ (b) e $3 \%$ (c). Nota-se que os filmes à base de fécula de batata não apresentaram superfície rugosa, o que está de acordo com as observações de Bengtsson et al. (2003) que utilizaram a microscopia eletrônica de varredura no monitoramento da superfície de filmes de amido de batata.

Pela Figura 3, apresentam-se as fotomicrografias dos filmes de fécula de mandioca nas concentrações de $1 \%$ (a), $2 \%$ (b) e $3 \%$ (c).

Nas Figuras 3(a), 3(b) e 3(c) nota-se que não ocorreu formação de trincas ou falhas na superfície dos filmes. Os filmes apresentaram superfície bastante homogênea e lisa. O que se pode observar é que FFM1 apresentou a superfície aparentemente diferente em relação a FFM2 e FFM3. Observam-se pelas fotomicrografias de FAM, FFB e FFM, que estes últimos apresentam uma superfície muito mais lisa e homogênea quando comparados a FAM e FFB, principalmente o FFM2. Isso pode ser devido principalmente à temperatura de elaboração dos géis que provavelmente foi suficiente para a completa gelatinização da fécula de mandioca contribuindo para a formação de uma superfície mais homogênea dos filmes, ou seja, a temperatura empregada de $70^{\circ} \mathrm{C}$ foi superior à temperatura de gelatinização da fécula de mandioca $\left(56\right.$ a $\left.62^{\circ} \mathrm{C}\right)$. 


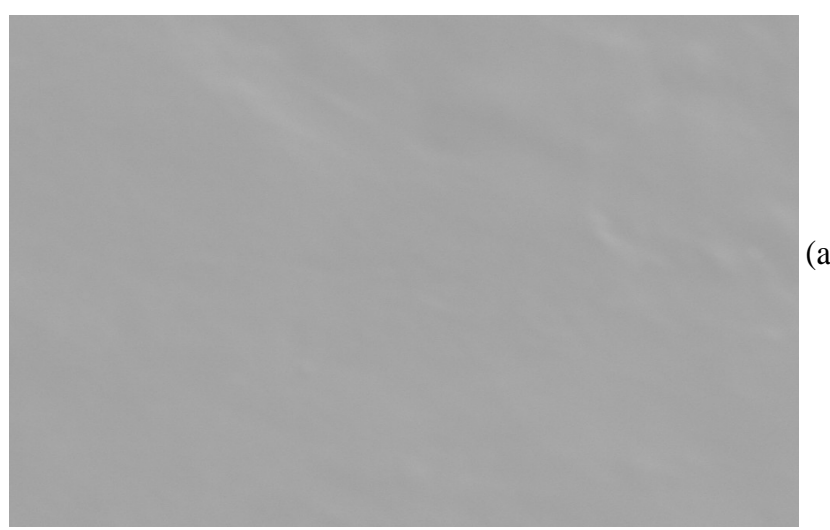

(a)

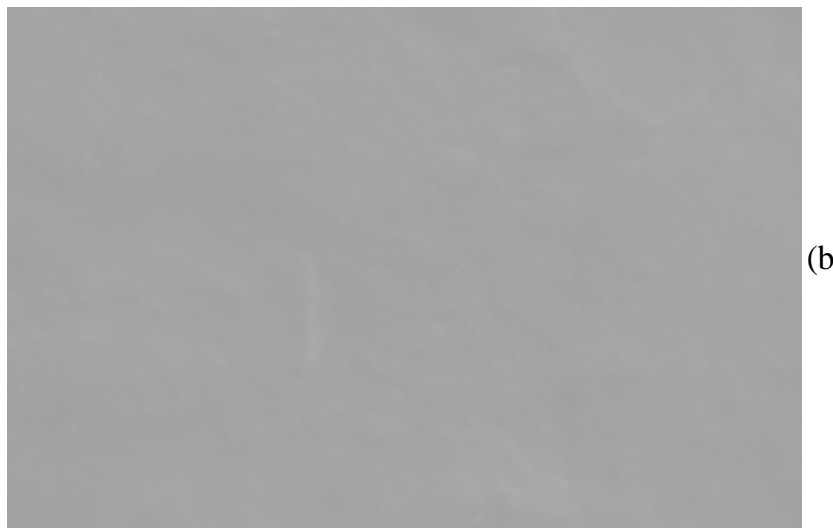

(b)



FIGURA 2 - Fotomicrografias de biofilmes de fécula de batata (FFB) nas concentrações de 1\% (a), 2\% (b) e 3\% (c). 


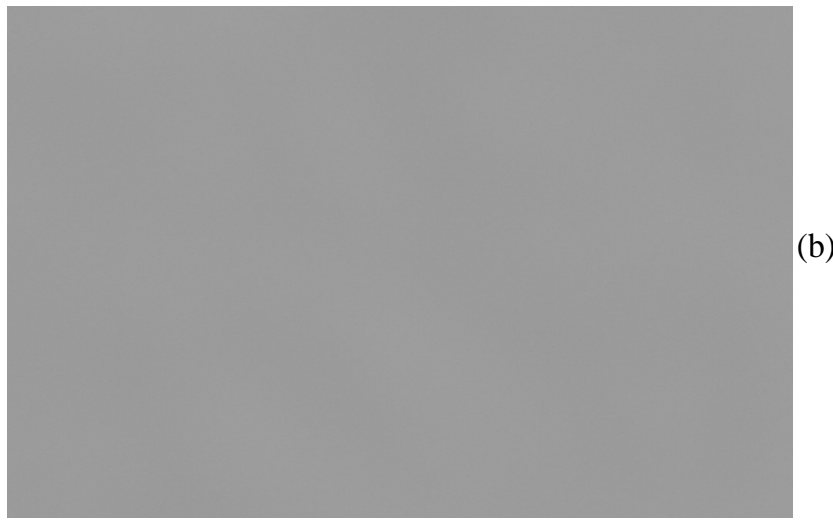

(c)

FIGURA 3 - Fotomicrografias de biofilmes de fécula de mandioca (FFM) nas concentrações de 1\% (a), 2\% (b) e 3\% (c). 


\section{Ângulo de contato}

A análise de regressão foi significativa para o ângulo de contato (Tabela 1). Na Figura 4, apresenta-se o comportamento das médias estimadas para o ângulo de contato e as equações de regressão para FAM, FFB e FFM nas concentrações de 1,2 e $3 \%$.

Para filmes de amido de milho não houve efeito significativo da concentração de amido sobre o ângulo de contato, sendo o valor descrito pela média (Figura 4). Observa-se na Figura 4 que o ângulo de contato para filmes de fécula de batata e filmes de fécula de mandioca diminuiu com o aumento da concentração de amido. $O$ ângulo de contato para FFB a $3 \%$ foi de aproximadamente $41^{\circ}$. De acordo com Mali et al. (2004), uma maior concentração de amido proporciona uma matriz amilácea com maior número de grupos hidrofílicos, ou seja, haveria maior interação desses grupos com a água. Logo, observa-se que o ângulo de contato da água com a superfície dos filmes diminuiu o que poderia estar relacionado à interação da gota de água com a superfície do filme, conseqüentemente menor ângulo de contato.
Para Thiré et al. (2004), os plásticos de amido, apresentam a característica de hidrofilicidade o que pode limitar sua aplicação tecnológica. Os filmes absorvem água facilmente e se incham o que pode resultar em perda nas suas propriedades mecânicas, entre outras (HULLEMAN et al., 1998; RINDLAV-WESTLING et al., 1998; THIRÉ et al., 2004). Bialopiotrowicz (2003) analisou o ângulo de contato para diferentes líquidos (água, glicerol, formamida, etileno glicol, diiodometano e polimetila metacrilato) em filmes à base de amido de milho e fécula de batata em diferentes concentrações (2, $4,6,8,10$ e $12 \%$ ) e observou que para os filmes de amido de milho, o ângulo de contato diminuiu linearmente com o aumento da concentração de amido e que os filmes à base de fécula de batata apresentaram valores para o ângulo de contato maior em relação aos filmes de amido de milho, mas também decresceram linearmente com o aumento da concentração de fécula. O autor lembra ainda, que após a secagem a água se liga à cadeia polimérica do amido e não fica livre sobre a superfície do filme.

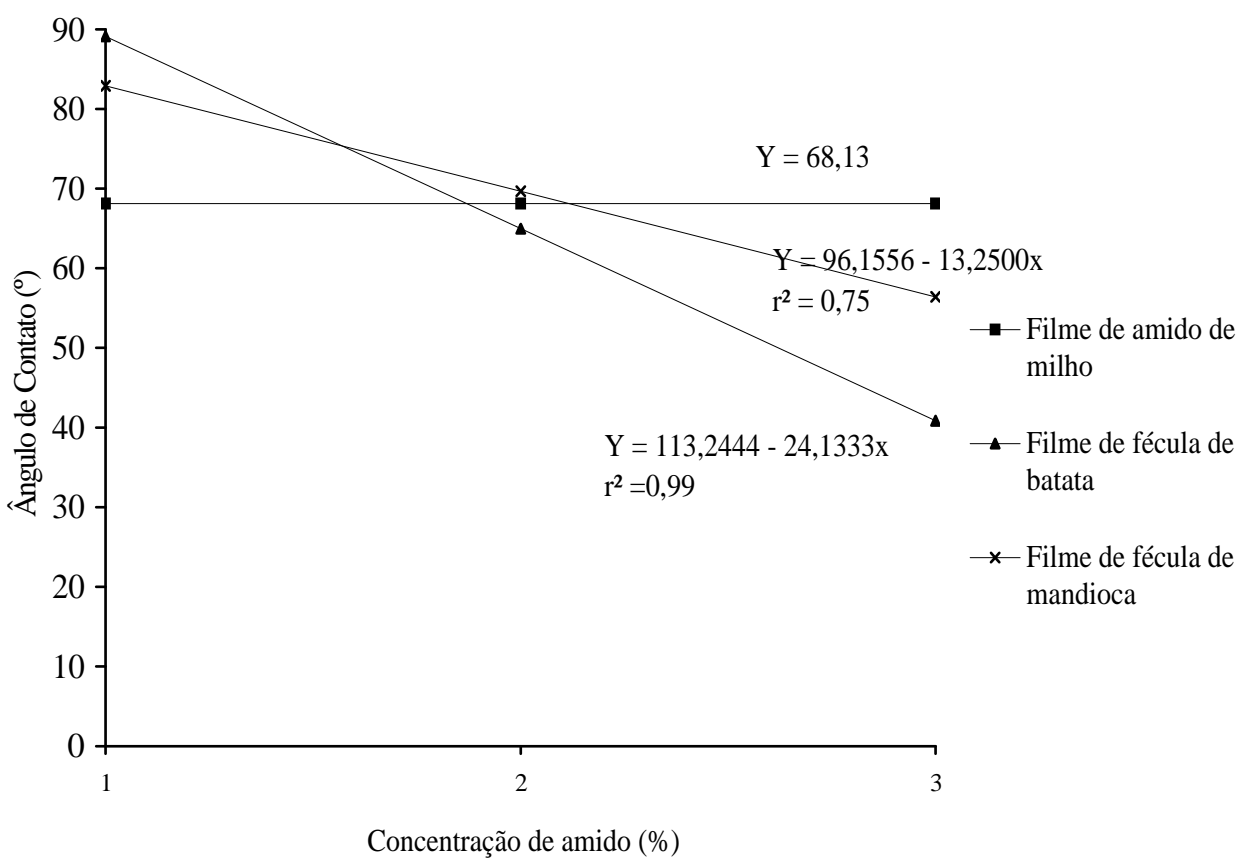

FIGURA 4 - Representação gráfica e equações de regressão para o ângulo de contato $\left(^{\circ}\right)$ dos filmes de amido de milho (FAM), filmes de fécula de batata (FFB) e filmes de fécula de mandioca (FFM) em função da concentração de amido (\%). 


\section{CONCLUSÕES}

Pode-se concluir que a concentração e o tipo de amido influenciaram as propriedades dos biofilmes analisadas. Biofilmes elaborados com fécula de mandioca apresentaram maior diferença de cor em relação aos biofilmes de amido de milho e fécula de batata. Quanto às fotomicrografias dos biofilmes, os de fécula de batata apresentaram a superfície topográfica mais homogênea em relação aos biofilmes de fécula de mandioca e amido de milho. Para biofilmes de fécula de mandioca e batata, há uma correlação linear entre o ângulo de contato e o teor de amido, ou seja, maior teor de amido menor o ângulo de contato, enquanto que para os biofilmes de amido de milho esta relação não foi observada.

\section{REFERÊNCIAS BIBLIOGRÁFICAS}

ABURTO, J.; THICBAUD, S.; ALRIE, I.; BORREDON, E.; BIKIARIS, D.; PRINOS, J.; PANAYIOTOU, C. Properties of octanoated starch and its blends with polyethylene. Carbohydrate Polymers, [S.1.], v. 34, n. 1/2, p. 101-112, 1999.

BENGTSSON, M.; KOCH, K.; GATENHOLM, P. Surface octanoylation of high-amylose potato starch films. Carbohydrate Polymers, [S.1.], v. 54, p. 1-11, 2003. Disponível em: <http://Www.elsevier.com/carbpol’. Acesso em: 6 jan. 2004.

BIALOPIOTROWICZ, T. Wettability of starch gel films. Food Hydrocolloids, [S.1.], v. 17, p. 141-147, 2003.

BRUNO, F. F.; AKKARA, J. A. A.; AYYAGARI, M.; KAPLAN, D. L.; GROSS, R.; SWIFT, G.; DORDICK, J. S. Enzymatic modification of insoluble amylose in organic solvents. Macromolecules, [S.1.], v. 28, n. 26, p. 8881-8883, 1995.

CEREDA, M. P.; BERTOLINI, A.; EVANGELISTA, R. M. Uso de amido em substituição às cêras na elaboração de películas na conservação pós-colheita de frutas e hortaliças. In: CONGRESSO BRASILEIRO DE MANDIOCA, 7., 1992, Recife. Anais... Recife: [s.n.], 1992. p. 107.

CEREDA, M. P.; CASTRO, T. M. R. de; HENRIQUE, C. M. Embalagens de materiais biodegradáveis. In: WORKSHOP SOBRE TECNOLOGIAS EM AGROINDÚSTRIAS DE TUBEROSAS TROPICAIS, 1., 2003, Botucatu. Anais... Botucatu: CERAT/UNESP, 2003. p. 124.
CIACCO, C. F.; CRUZ, R. Fabricação do amido e sua utilização. São Paulo: Secretaria da Indústria, Comércio, Ciência e Tecnologia, [1982]. 152 p.

FERREIRA, D. F. Sisvar versão 4.3. Lavras: DEX-UFLA, 2003.

GENNADIOS, A.; WELLER, C. L.; HANNA, M. A.; FRONING, G. W. Mechanical and barrier properties of egg albumen films. Journal of Food Science, Chicago, v. 61, p. 585-589, 1996.

HULLEMAN, S.; JANSSEN, F.; FEIL, I. The role of water during plastification of native starches. Polymer, [S.1.], v. 39, p. 2043-2048, 1998.

JANSSON, A.; THUVANDER, F. Influence of thickness on the mechanical properties for starch films. Carbohydrate polymers, [S.1.], v. 56, p. 499-503, 2004.

JAYASEKARA, R.; HARDING, I.; BOWATER, I.; CHRISTIE, G. B. Y.; LONERGAN, G. T. Preparation, surface modification and characterization of solution cast starch PVA blended films. Polymer Testing, [S.1.], v. 23, p. 17-27, 2004.

LAROTONDA, F. D. S.; MATSUI, K. N.; SOLDI, V.; LAURINDO, J. B. Biodegradable films made from raw and acetylated cassava starch. Brazilian Archives of Biology and Technology, Curitiba, v. 47, n. 3, p. 477-484, July 2004.

LAWTON, L. W. Effect of starch type on the properties of starch containing films. Carbohydrate Polymers, [S.l.], v. 29, p. 203-208, 1996.

MALI, S.; GROSSMANN, M. V. E.; GARCÍA, M. A.; MARTINO, M. N.; ZARITZKY, N. E. Barrier, mechanical and optical properties of plasticized yam starch films. Carbohydrate Polymers, [S.1.], v. 56, p. 129-135, 2004. Disponível em: $\langle$ hhttp://Www.sciencedirect.com خ. Acesso em: 20 maio 2004.

MARTINS, J. Amigo da pressa. Ciência Hoje, Rio de Janeiro, v. 33, n. 196, p. 42-43, ago. 2003.

OLIVEIRA, M. A.; CEREDA, M. P. Efeito da película de mandioca na conservação de goiabas. Brazilian Journal of Food Technology, [S.1.], v. 2, n. 1/2, p. 97-102, maio 1999. 
POOVARODOM, N.; PRADITDOUNG, S. The development of biodegradable packages from Cassava starch. Thailand: Kasetsart University, 2003.

RINDLAV-WESTLING, A.; STANDING, M.; HERMANSSON, A.; GATENHOLM, P. Structure, mechanical and barrier properties of amylose and amilopectin films. Carbohydrate Polymers, [S.1.], v. 36, p. 217-224, 1998.

ROY, S.; WELLER, C. L.; GENNADIOS, A.; ZEECE, M. G.; TESTIN, R. F. Physical and molecular properties of wheat gluten films cast from heated film-forming solutions. Journal of Food Science, Chicago, v. 64, n. 1, p. 57-60, 1999.

SHANDERA, D. L.; JACKSON, D. S. Corn kernel structural integrity: analisys using solvent and heat treatments. Cereal Chemistry, Saint Paul, v. 79, n. 2, p. 308-316, Nov. 2002.

SOBRAL, P. J. do A. Influência da espessura de biofilmes feitos à base de proteínas miofibrilares sobre suas propriedades funcionais. Pesquisa Agropecuária Brasileira, Brasília, v. 35, n. 6, p. 1251-1259, jan. 2000.

THIRÉ, R. M. S. M.; SIMÃO, R. A.; ANDRADE, C. T. High resolution imaging of the microstructure of maize starch films. Carbohydrate Polymers, [S.1.], v. 54, p. 149-158, 2003. Disponível em: <http://www.elsevier.com/locate/carbpol Acesso em: 20 jan. 2004.

THIRÉ, R. M. S. M.; SIMÃO, R. A.; ARAÚJO, P. J. G.; ACHETE, C. A.; ANDRADE, C. T. de. Redução da hidrofilicidade de filmes biodegradáveis à base de amido por meio de polimerização por plasma. Polímeros: Ciência e Tecnologia, [S.1.], v. 14, n. 1, p. 57-62, 2004.

WATANABE, A.; LARSSON, H.; ELIASSON, A. C. Effect of physical state of nonpolar lipids on rheology and microstructure of gluten-starch and wheat flour doughs. Cereal Chemistry, Saint Paul, v. 79, n. 2, p. 203-209, 2002.

WILHELM, H. M.; SIERAKOWSKI, M. R.; SOUZA, G. P.; WYPYCH, F. Starch films reinforced with mineral clay. Carbohydrate Polymers, [S.1.], v. 52, p. 101-110, 2003. 